\title{
Nivel de madurez para la gestión de la innovación en el sector eléctrico: caso de investigación de campo aplicado en Costa Rica
}

\author{
William Gutiérrez-Sandíl \\ Santos Gracia-Villar² \\ Luis Alonso Dzul-López ${ }^{3}$ \\ Federico Fernández Díez ${ }^{4}$
}

Fecha de recepción: 25 de marzo del 2011 Fecha de aceptación:30 de setiembre del 2011

Gutiérrez, W; Gracia, S; Dzul, L; Fernández, F. Nivel de madurez para la gestión de la innovación en el sector eléctrico: caso de investigación de

campo aplicado en Costa Rica. Tecnología en Marcha. Vol. 25, № 2. Abril-Junio 2012. Pág 87-97.

Máster en Administración de Proyectos. Ingeniero Electrónico. Innprotec Education Group, Heredia, Costa Rica. Teléfono: 8377 73 03. Correo electrónico: wgutierrezs@hotmail.com

2 Ingeniero industrial. Universidad Latinoamericana de Ciencia y Tecnología. Sede Metropolitana Urbanización Tournón. Teléfono: 2523-4000. Correo electrónico: santos@funiber.org Ingeniero civil. Fundación Universitaria Iberoamericana. Paseo García Faria, 29 - 08005 Barcelona. Teléfono: 54|| 438 |865I. Correo electrónico: luis.alonso.dzul@upc.edu

4 Doctor en Ciencias de la educación. Investigador en el Departamento de Proyectos de Ingeniería, Universidad Politécnica de Cataluña. Correo electrónico: federico.fernandez@upc.edu 


\section{Resumen}

Los nuevos modelos económicos provocan que las organizaciones deban responder de manera rápida a los diferentes cambios que generan los actores y el mismo entorno en los mercados. La gestión de las iniciativas de innovación por medio de proyectos se convierte en una útil herramienta de gestión empresarial. No obstante, cabe la pregunta sobre cuál será el grado de madurez con el que las organizaciones realizan dichas gestiones.

Para ello, el Modelo de Madurez La Pirámide Invertida (MMPI) constituye una herramienta con la cual es posible valorar la gestión estructurada y sistemática del proceso innovador en la organización.

Dicha herramienta fue aplicada al sector de distribución de Energía Eléctrica en Costa Rica, Latinoamérica. Mediante la aplicación de una investigación exploratoria, se documentó el estado actual de algunas organizaciones del sector que sirven como referencia para realizar un análisis del mismo. El método científico fue la guía metodológica para el planteamiento y desarrollo de la presente investigación.

Los resultados obtenidos reflejan una realidad latente en muchas organizaciones de este y otros sectores productivos. A partir de este primer esfuerzo será posible conocer cuál es esa realidad y las medidas que deben ser tomadas para poder contar con sectores productivas más eficientes y organizaciones que generen mayor valor.

\section{Palabras clave}

Gestión de la innovación, modelo de madurez la pirámide invertida, gestión de proyectos, estrategia empresarial, modelos de madurez, sector eléctrico, investigación, distribución eléctrica.

\section{Abstract}

The new economic models that an organization must lead quickly to respond to the various changes that generate the actors and the same environment in the markets. The management of innovation initiatives through projects becomes a useful tool for business management. However, the question arises as to what will be the degree of maturity that organizations engaged in these efforts.

This Maturity Model Inverted Pyramid (MMPI) is a tool with which it is possible to evaluate the structured and systematic management of the innovation process in the organization.

This tool was applied to the distribution sector of Electricity in Costa Rica, Latin America. Through the application of exploratory research, documented the current state of some industry organizations that serve as reference for an analysis of it. The scientific method was the methodological guide for the planning and development of this research.

The results show a latent reality in many organizations in this and other productive sectors. From this first effort will be possible to know what that reality and measures must be taken in order to have more efficient and productive sectors that generate higher value organizations.

\section{Key words}

Innovation management, maturity model inverted pyramid, project management, business strategy, maturity models, electrical sector, research, electrical distribution. 


\section{Introducción}

Las diferentes áreas del conocimiento han abordado al tema de la innovación desde varias perspectivas, así como se ha hecho con la gestión del cambio y del conocimiento. De igual manera, se han desarrollado metodologías para llevar a cabo la gestión de la innovación y la tecnología en las organizaciones.

El problema medular radica en la carencia de un modelo de este tipo, lo que provoca que las organizaciones no cuenten con un patrón de referencia contra el cual contrastar sus avances, tal y como ocurre con modelos ya establecidos para gestión de proyectos, gestión del conocimiento, gestión del cambio y gestión de la calidad.

¿Cuál es el nivel de madurez con respecto a la gestión de la innovación en el sector de distribución de energía eléctrica en Costa Rica en el periodo 2007-2008?

Para responder esta interrogante, se parte del planteamiento de la siguiente hipótesis: El sector de distribución de energía eléctrica en Costa Rica se encuentra en etapas tempranas de madurez con respecto a la gestión de la innovación, debido a que no cuenta con sistematización de sus procesos innovadores.

Esta es la primera investigación en la que se midió el grado de sistematización con que las compañías del sector de distribución eléctrica han realizado su gestión en relación con los procesos de innovación. Fue desarrollada en Costa Rica, en el 2008.

\section{Materiales y métodos}

Cuando se realiza una investigación acerca de una empresa o sector de la producción, se le considera como práctica ya que, según cita Bernal (2000), se genera información que puede ser utilizada para generar directrices tendientes a mejorar la gestión de dicha empresa o sector productivo. Para este trabajo de investigación se utilizó el método científico (modelo general), tomado de Muñoz (1998). Este es un conjunto de etapas y actividades que señalan el procedimiento para ejecutar una investigación, cuyos resultados son aprobados y validados por la comunidad científica. Las etapas de este método fueron seis y se detallan a continuación:
Etapa I. Planteamiento del problema

En esta etapa se buscó plantear el problema de investigación. Para ello se debe enunciar el problema, después se formula de manera explícita cuáles serían los componentes del problema de investigación.

\section{Etapa II. Formulación de la hipótesis}

Para este apartado se realiza el planteamiento de la hipótesis. Para ello se debe definir la interrogante sobre la cual se plantea la hipótesis. Una vez definido dicho elemento se procede a realizar la formulación de la hipótesis.

\section{Etapa III. Levantamiento de la información}

Recolección de datos de fuentes primarias. El objetivo era medir el grado de conformidad y nivel de madurez con respecto a los procesos de innovación de las empresas de distribución de energía eléctrica en Costa Rica en el 2007. Una vez que se definieron las organizaciones que representarían a la población estadística, se visitó cada organización para recabar la información inicial para diseñar el perfil de la empresa, y ver que todas las seleccionadas fueran representativas de la población por evaluar.

Se realizaron entrevistas estructuradas y abiertas de manera presencial o vía telefónica, así como la aplicación de instrumentos de evaluación, los cuales se utilizaron para recabar datos que permitieron determinar el grado de madurez o sistematización con que realizan la gestión de la innovación, las fuentes de generación de las innovaciones, los factores que impulsan a los sistemas de innovación y cómo toda esta gestión de las innovaciones afecta a la gestión empresarial.

Recopilación de datos de fuentes secundarias. Para ello se utilizó Internet como principal fuente. Además, la revisión bibliográfica ayudó al investigador a conocer el estado del arte al momento de la investigación para definir líneas de pensamiento y de análisis requeridas para valorar la gestión de los procesos innovadores en el sector de distribución de energía eléctrica en Costa Rica.

Instrumentos de medición del grado de madurez: Se emplearon los siguientes instrumentos durante el desarrollo de la investigación:

a) Entrevista. La cual fue aplica en dos modalidades. Una entrevista semi estructurada realizada a los individuos sujetos de prueba que 
representan a las organizaciones en estudio, la cual se plantea de forma abierta, con un hilo conductor o como una conversación de preguntas puntuales. La segunda, una entrevista focalizada, la cual buscó resolver dudas generadas en los resultados de los instrumentos de medición aplicados.

b) Seminario - taller. Para realizar el estudio se impartió un seminario- taller en cuanto al uso del Instrumento de medición del MMPI con dos finalidades:

- Uniformar en todos los sujetos de prueba el concepto y alcance de la palabra "innovación" para que cuenten con una misma base conceptual y con ello uniformar la medición.

- Transmitir a los sujetos evaluadores de las unidades de prueba la filosofía y metodología que utiliza el MMPI a la hora de realizar el proceso de valoración.

c) Test I. Valoración utilizando la herramienta SOFT-INNOVA. Se utilizó el Instrumento I del Modelo de la Pirámide Invertida, el cual permite determinar el estado actual de los procesos de innovación. Para ello, se desarrolló un instrumento de software que facilita la recolección de los datos en campo. Además, las unidades de prueba suministraron debidamente lleno el instrumento de medición, que contiene un set de indicadores relacionados con la gestión de la innovación y la gestión empresarial. Asimismo, se utilizó el tercer instrumento denominado Catálogo de Innovaciones, mediante el cual se permite cuantificar la cantidad de innovaciones sobresalientes de la organización y observar los efectos de estas en la gestión empresarial del periodo de medición.

d) Presentación de resultados. Corresponde a la presentación de resultados del Estado del sector eléctrico en Costa Rica, a partir de los datos recabados en las mediciones aplicadas a la muestra.

Aplicación de los instrumentos de medición. Para la actividad se estableció contacto con cada organización. Se seleccionó una persona que sirvió como contacto en la unidad de prueba. La selección se realizó con base en el perfil y grado de relación de los individuos cada uno de los sub-bloques que evalúa el MMPI. El grupo varió entre 5 y 20 personas por cada unidad de prueba. Se recomendó el número de 15 personas titulares, uno por cada sub-bloque a evaluar. Para realizar la medición en el instrumento SOFT-INNOVA los individuos de cada unidad de prueba contaron con un tiempo que oscila entre 1.5 a 3.5 horas, dependiente del sub-bloque que se encontraba en evaluación y el individuo que realizaba la acción.

El catálogo de Innovaciones fue completado por las personas participantes en el seminario-taller I de acuerdo con las especificaciones dadas durante el mismo. Para esta tarea los participantes cuentan con un periodo de tiempo de entre 4 a 8 horas. Luego, todas las respuestas se consolidaron en un solo documento. A cada organización se le entregó un instrumento en el cual se encuentra el set de indicadores. El responsable de remitirlo debidamente contestado era la persona contacto en cada unidad de prueba. Para realizarlo, el individuo contacto contó con 8 días de tiempo a partir de la fecha de su recepción.

\section{Etapa IV.Análisis de datos}

Procesamiento y análisis de los datos. Luego de aplicar los instrumentos, la información se procesa para que sea accesible.

- El nivel de madurez actual de manera separada de cada una de las unidades de prueba.

- $\quad$ El comportamiento del sector en cuanto al nivel de madurez con respecto a la gestión de la innovación.

- $\quad$ Las relaciones en cuanto a la gestión de las funciones, variables y proceso que realiza cada unidad de prueba y del sector con respecto a la gestión de sus sistemas de innovación.

- La procedencia de las innovaciones que desarrolla cada unidad de prueba, su frecuencia en la gestión empresarial de cada unidad de prueba.

Los datos obtenidos permitieron obtener información variada e importante para realizar ajustes en el proceso de gestión de la innovación como tal, tanto como para identificar las áreas de la organización que requerían procesos de mejora, realizados por medio de proyectos innovadores para aumentar la eficiencia de la gestión empresarial de la innovación; ello contribuyó con las organizaciones para identificar sus oportunidades de mejora y sus puntos de ajuste. 
Cuadro I. Empresas de Distribución de energía eléctrica que operan en el mercado de Costa Rica

\begin{tabular}{|c|c|}
\hline Empresa & Categoría \\
\hline ICE & Autónoma \\
\hline CNFL & Autónoma \\
\hline ESPH SA & Sociedad anónima municipal \\
\hline Jasec & Cooperativa mediana \\
\hline C. Guanacaste & Cooperativa mediana \\
\hline C. Coopelesca & Cooperativa pequeña \\
\hline C. Santos & Cooperativa pequeña \\
\hline C. Alfaro R. &
\end{tabular}

Fuente: CENPE (2006) Costa Rica sector eléctrico nacional. Datos demanda al 2006.

Cuadro 2. Resultados de la medición a nivel de bloques para la población del sector eléctrico en Costa Rica

\begin{tabular}{|c|c|c|c|c|}
\hline Nivel de las organizaciones & $\begin{array}{c}\text { Cooperativa } \\
\text { mediana }\end{array}$ & $\begin{array}{c}\text { Cooperativa } \\
\text { pequeña }\end{array}$ & $\begin{array}{c}\text { S.A. } \\
\text { municipal }\end{array}$ & $\begin{array}{c}\text { Sector } \\
\text { eléctrico }\end{array}$ \\
\hline Grado de conformidad & 2,02 & 2,84 & 1,20 & 1,57 \\
\hline Nivel de madurez & Principiante & Principiante & Aprendiz & Aprendiz \\
\hline
\end{tabular}

Fuente. Elaboración propia. Medición de campo en Coopelesca (2008), ESPH S.A. (2008), Coopealfaroruiz (2008).

Cuadro 3.a. Instrumentos estadísticos para análisis de resultados para unidades de prueba

\begin{tabular}{|c|c|c|c|c|c|c|}
\hline Organización & Ponderación & $\begin{array}{c}\text { Promedio } \\
\text { (grado de } \\
\text { conformidad) }\end{array}$ & $\begin{array}{c}\text { StdDev of } \\
\text { calificación }\end{array}$ & $\begin{array}{c}\text { Máx de } \\
\text { calificación }\end{array}$ & $\begin{array}{c}\text { Mín de } \\
\text { calificación }\end{array}$ & $\begin{array}{c}\text { Var de } \\
\text { calificación } \\
\text { muestra }\end{array}$ \\
\hline Cooperativa mediana & $40,90 \%$ & 2,02 & 1,17 & 7 & 1 & 0,23 \\
\hline Cooperativa pequeña & $1,92 \%$ & 2,84 & 1,69 & 7 & 1 & 0,00 \\
\hline S.A. municipal & $57,18 \%$ & 1,20 & 0,69 & 6 & 1 & 0,16 \\
\hline Sector eléctrico & $100,00 \%$ & 1,57 & 0,69 & 6,3706 & 1 \\
\hline
\end{tabular}

Cuadro 3.b. Instrumentos estadísticos para análisis de resultados para unidades de prueba

\begin{tabular}{|c|c|c|c|c|c|c|c|c|}
\hline \multicolumn{10}{|c|}{ Frecuencia reportada en el grado de conformidad } \\
\hline Organización & 1 & 2 & 3 & 4 & 5 & 6 & 7 & 8 \\
\hline Cooperativa mediana & $6.102,00$ & $3.094,00$ & $2.366,00$ & $1.254,00$ & 409,00 & 42,00 & 13,00 & 0,00 \\
\hline Cooperativa pequeña & $4.829,00$ & $1.003,00$ & $2.692,00$ & $1.961,00$ & $1.963,00$ & 720,00 & 112,00 & 0,00 \\
\hline S.A. municipal & $11.918,0$ & 582,00 & 353,00 & 307,00 & 98,00 & 22,00 & 0,00 & 0,00 \\
\hline Sector eléctrico & $2.248,00$ & 18,00 & 18,00 & 46,00 & 7,00 & 0,00 & 0,00 & 0,00 \\
\hline
\end{tabular}

Fuente. Elaboración propia. Medición de campo en Coopelesca (2008), ESPH S.A. (2008), Coopealfaroruiz (2008). 
Cuadro 4. Clasificación de las Innovaciones para las unidades de muestra

\begin{tabular}{|c|c|c|c|c|c|c|c|c|}
\hline \multirow{2}{*}{$\begin{array}{c}\text { Unidad de } \\
\text { muestra }\end{array}$} & \multicolumn{2}{|c|}{ S.A. municipal } & \multicolumn{2}{|c|}{ Cooperativa pequeña } & \multicolumn{2}{|c|}{ Cooperativa mediana } & \multicolumn{2}{|c|}{$\begin{array}{l}\text { Resultados total de la } \\
\text { muestra }\end{array}$} \\
\hline & $\begin{array}{l}\text { Cantidad } \\
\text { S.A. } \\
\text { municipal }\end{array}$ & $\begin{array}{l}\text { Porcentaje } \\
\text { de } \\
\text { participación } \\
\text { por } \\
\text { categoría }\end{array}$ & $\begin{array}{c}\text { Cantidad } \\
\text { cooperativa } \\
\text { pequeña }\end{array}$ & $\begin{array}{l}\text { Distribución } \\
\text { porcentual }\end{array}$ & $\begin{array}{c}\text { Cantidad } \\
\text { cooperativa } \\
\text { mediana }\end{array}$ & $\begin{array}{c}\text { Distribución } \\
\text { porcentual }\end{array}$ & $\begin{array}{l}\text { Cantidad } \\
\text { total de } \\
\text { iniciativa }\end{array}$ & $\begin{array}{c}\text { Distribución } \\
\text { porcentual }\end{array}$ \\
\hline Cobertura & 59 & $100,00 \%$ & 4 & $100 \%$ & 22 & $100 \%$ & 85 & $100 \%$ \\
\hline Empresa & 47 & $79,66 \%$ & 4 & $100 \%$ & 19 & $86 \%$ & 70 & $82 \%$ \\
\hline Región / país & 12 & $20,34 \%$ & 0 & $0 \%$ & 3 & $14 \%$ & 15 & $18 \%$ \\
\hline Global & 0 & $0,00 \%$ & 0 & $0 \%$ & 0 & $0 \%$ & 0 & $0 \%$ \\
\hline Naturaleza & 59 & $100,00 \%$ & 4 & $100 \%$ & 22 & $100 \%$ & 85 & $100 \%$ \\
\hline Proceso & 28 & $47,46 \%$ & I & $25 \%$ & 10 & $45 \%$ & 39 & $46 \%$ \\
\hline Mercado & 2 & $3,39 \%$ & 0 & $0 \%$ & I & $5 \%$ & 3 & $4 \%$ \\
\hline Organización & 10 & $16,95 \%$ & 0 & $0 \%$ & 9 & $41 \%$ & 19 & $22 \%$ \\
\hline Producto & 19 & $32,20 \%$ & 3 & $75 \%$ & 2 & $9 \%$ & 24 & $28 \%$ \\
\hline Grado & 59 & $100,00 \%$ & 4 & $100 \%$ & 22 & $100 \%$ & 85 & $100 \%$ \\
\hline Radical & 27 & $45,76 \%$ & 2 & $50 \%$ & 5 & $23 \%$ & 34 & $40 \%$ \\
\hline Incremental & 32 & $54,24 \%$ & 2 & $50 \%$ & 17 & $77 \%$ & 51 & $60 \%$ \\
\hline $\begin{array}{l}\text { Total por } \\
\text { unidad de } \\
\text { muestra }\end{array}$ & 59 & $100 \%$ & 4 & $100 \%$ & 22 & $100 \%$ & 85 & $100 \%$ \\
\hline
\end{tabular}

Fuente. Elaboración propia. Medición de campo en Coopelesca (2008), ESPH SA (2008), Coopealfaroruiz (2008).

Propuesta de estructura e implementación. La investigación realizada generó un resultado en el que se muestra el estado actual de cada sujeto de estudio, la estimación del nivel de madurez en la gestión de la Innovación en el sector aplicado, para la zona geográfica cubierta, en este caso Costa Rica. Las evaluaciones aplicadas suministran información para posibles oportunidades de mejora que deben ser desarrolladas, señala las funciones de la gestión de innovación que deberían ser mejoradas y los elementos (unidades, procesos funcionales) que requieren de una mejora en su nivel de gestión para equilibrar a los otros de su nivel y mejorar la gestión operativa en dichos elementos, los cuales influirán en el desempeño general de la Gestión Empresarial.

\section{Etapa V. Comprobación de la hipótesis}

Para realizar dicha acción se desarrollaron las acciones de seminario-taller I para las unidades de prueba. Lo anterior, a partir del uso de la herramienta SOFTINNOVA, catálogo de innovaciones e indicadores de gestión.

\section{Resultados y discusión}

Los instrumentos se aplicaron a los sujetos de estudio (las organizaciones). Se seleccionaron tres unidades de prueba, las cuales son una representación del sector por medir. En el cuadrol se muestran las unidades de prueba seleccionadas:

La medición fue realizada en una sociedad anónima municipal, una cooperativa de pequeño tamaño y una de mediano tamaño. Los niveles de madurez y grados de conformidad en el desarrollo de las acciones innovadoras encontrados se muestran en el cuadro 2.

En el cuadro 3.a se muestran los pesos de las unidades de medición. La desviación estándar sobre la unidad de muestra presenta un valor máximo de 1,69. Los resultados inferidos con relación a la desviación estándar tienen un punto importante; es el hecho de que la muestra utilizada son solamente tres unidades, lo cual hace que las variaciones que se presenten entre los datos generan un impacto fuerte en los resultados de la ponderación. 
En el cuadro 4 se encuentra el consolidado de las innovaciones producidas por las distintas unidades de prueba para el periodo bajo estudio; dicha clasificación de acuerdo con la tipificación que realiza el MMPI en su Cubo de la Innovación, las iniciativas innovadoras pueden clasificarse por cobertura, naturaleza y grado. Esta clasificación para las innovaciones registradas tiene como horizonte temporal el periodo de medición bajo estudio (2007).

\section{Conclusiones sobre los resultados}

Todo el proceso creativo que fue desarrollado a lo largo de la investigación, inició con el levantamiento de información para crear un modelo propio, original, por medio del cual fuese posible parametrizar la gestión de una organización, precisar cuáles son las áreas de mejora y utilizar la innovación el medio, la herramienta, el gestor de los recursos en la organización. A partir de ese concepto se inició la creación del Modelo de Madurez La Pirámide Invertida (MMPI) desarrollado por Gutiérrez (2007). En este apartado se expondrán los efectos de los resultados obtenidos, así como posibles fuentes o acciones que han intervenido para la obtención de los resultados recopilados.

Grado de madurez en la gestión de las innovaciones en el sector eléctrico

Las unidades de medición del sector de distribución de energía eléctrica en Costa Rica se encuentran en etapas tempranas, con respecto a la sistematización de sus sistemas, proyectos e iniciativas para la innovación. Los desarrollos se han realizado tradicionalmente mediante esquemas ad-hoc y los grados de conformidad así como los niveles de sistematización en la gestión de las iniciativas de innovación son bajos.

Obsérvense los resultados de el cuadro 2, donde se muestra que, de acuerdo con los parámetros de medición del MMPI, las organizaciones cuentan con grados de conformidad entre 2, 84 y 1,20. Estos resultados ubican a las organizaciones en niveles de madurez de principiante y aprendiz.

El punto medular recae sobre el hecho de que las organizaciones del sector en general, no se han preocupado o por lo menos no lo han demostrado abiertamente en la evidencia recabada y valorada durante la intervención de campo de la presente investigación; de contar con una gestión sistemática de sus iniciativas y proyectos de innovación. Más aún, no cuentan con un sistema formal para la gestión de los ciclos de innovación en el momento de desarrollar y gestionar las iniciativas dentro de la organización, así como una organización de los procesos de innovación (Bravo, 2009).

Ante tal situación, el resultado obtenido es acorde con los parámetros de medición que establecen los diferentes grados de conformidad para los niveles de madurez. Las organizaciones deben entender que toda acción que se quiera llevar a un nivel de mejora o sistematización está obligada a pasar por un proceso de repetición y obtención de resultados sostenido, esto quiere decir, que debe ser planificado, iniciado, diseñado, ejecutado, controlado y concluido siguiendo una misma base metodológica. Con ello, la organización podrá prospectar sobre desarrollos futuros, con datos estandarizados y relacionables pues se encuentran bajo un mismo modelo de valoración.

\section{La innovación como pilar en la nueva economía}

La nueva economía establece cambios profundos en los esquemas de gerencia tradicionales para las organizaciones como lo comenta Kluyver (200 I). El conocimiento, uno de los pilares estructurales en el Modelo de Madurez La Pirámide Invertida (MMPI), se coloca como el elemento más importante sobre el cual se pueden construir los nuevos modelos empresariales, y el cambio como la constante sobre la cual deben las organizaciones plantear los nuevos escenarios.

Como lo expone Goodstein (1998) el uso de la Planificación Estratégica soportada sobre los procesos de innovación puede producir grandes mejoras en las organizaciones. Para ello, la Gestión de Proyectos será la herramienta principal para la puesta en marcha de las iniciativas de innovación. En dicho desarrollo, el MMPI suministra al desarrollador una serie de herramientas que han sido probadas por otras organizaciones y de las cuales se conoce su efectividad para ello, obsérvese COTEC (1998).

El uso de los principios de Proyectos, Innovación y Estrategia para el desarrollo de modelos empresariales, así como la implementación del MMPI no es garantía de éxito para ninguna organización. No obstante, sí es considerado como 
una verdad que las organizaciones que los adopten e implementen desde sus bases tendrán la capacidad de reaccionar de manera más rápida, consistente e innovadoramente en los cambiantes mercados de la economía actual.

La economía del conocimiento Funiber (2006) exige hoy a las empresas eléctricas, para el caso de esta investigación y para todas en general, tener sistemas de gestión de este valioso recurso; ya no solamente para poder llevar una gestión documental de las acciones y proyectos desarrollados, sino para establecer mecanismos de promoción de ideas y desarrollos creativos, en donde la cultura del cambio brinde a los colaboradores de la organización las herramientas requeridas para ser gestores de sus propias iniciativas, a la vez que colaboren como agentes en la transición y establecimiento de una cultura dispuesta a siempre aprender y enfrentar los diferentes cambios que el entorno les presente.

Será hasta en ese momento cuando se rompa el paradigma en la gestión empresarial y utilización de las herramientas para la innovación y, dichas compañías no se limitarán a esquemas tradicionales de pensamiento y desarrollo operativo.

\section{Efectividad de la gestión empresarial con} respecto a la innovación en el sector eléctrico en Costa Rica

Si se considera como único parámetro de referencia el grado de sistematización en la gestión de las innovaciones para definir qué tan eficiente es una organización, se tendría que decir que el sector de distribución de energía eléctrica en Costa Rica es bastante ineficiente. El sector de energía eléctrica es un negocio que hasta el 2010 ha sido un pseudo monopolio en Costa Rica. Las empresas distribuidoras han tenido mercados cautivos y sus necesidades de prospección comerciales no los han obligado a pensar en esquemas agresivos de competencia y superioridad de mercado.

Otro factor que ha influido en la efectividad de las acciones de gestión de las compañías eléctricas en este sector productivo, ha sido la pobre gestión documental y registro de estadísticas que presentan. $\mathrm{Si}$ recordamos las palabras de los doctores Kaplan y Norton (1992) haciendo referencia a la sistematización de un proceso empresarial "lo que no se mide no se controla y lo que no se controla no se mejora"; entonces podríamos extrapolar que parte del problema proviene de dicha fuente.

Para las organizaciones bajo estudio, no existió un registro previo de las necesidades o gestión de proyectos de línea innovadora antes de esta investigación; o dicho de otra manera, antes de la medición realizada en el 2008, producto de la presente investigación, no se había realizado un levantamiento y análisis formal de las iniciativas innovadoras que desarrollaban hasta ese momento, o que habían concluido en los doce meses anteriores a la medición. Por tanto, esto denota una falta de interés o desconocimiento del tema por parte de dichos actores del mercado sobre los beneficios que representa una eficiente gestión de las iniciativas y proyectos de innovación para la gestión estratégica de la organización, así como la maximización del recurso para obtener un mayor margen de beneficio.

En relación con los esquemas de gerencia que presentan las empresas del sector eléctrico en Costa Rica, y por conocimiento previo laboral de este mercado por parte de uno de los autores del presente documento, se tiene que las organizaciones bajo estudio, en general, no han dimensionado los beneficios que están dejando de percibir por gestionar sus organizaciones y proyectos basados en esquemas tradicionales de economías de costos; olvidan estos que la innovación canalizada y gestionada de manera eficiente, permite a dichas instituciones aumentar sus participaciones de mercado, así como, la calidad en el servicio lo cual deberá de trasladarse a una mayor satisfacción del cliente final con lo que se tendrá un mayor grado de fidelización del cliente en el mediano y largo plazo.

Las compañías del sector bajo estudio deben entender que la innovación conlleva una inversión, para el disfrute posterior del beneficio sobre el rendimiento de dicha inversión. La innovación no se debe entender como un algo más en mi producto, como podría inferirse de los resultados reportados por los sujetos bajo estudio en el capítulo cuarto de este documento. Por el contrario, deben entender que excelentes inversiones en proyectos claves de innovación representarán una carta de presentación atractiva para un mercado que migrará de esquemas tradicionales pseudo-monopólicos a mercados de competencia abierta. 
¿Qué se puede replicar en otros sectores productivos?

El aprender de las experiencias de éxito de otros agentes y sectores productivos es conveniente para la mejora de una gestión empresarial. En Latinoamérica es común el concepto de "tropicalización", como la observación e instauración organizacional de alguna tecnología observada y que podría representar algún beneficio para la organización. No obstante, es importante realizar una salvaguarda ante dicha situación. Transferir es diferente a copiar un modelo, y tropicalizar implica aún más allá, pues nos lleva al concepto de adaptar.

Estructura jerárquica ligera. Una de las buenas prácticas que es posible reproducir en diferentes organizaciones, sin importar el sector, y que ayudan a desarrollar una más ágil gestión de las iniciativas de innovación es el contar con una estructura jerárquica ligera o relativamente plana, eso significa que para la toma de las decisiones la comunicación entre los agentes involucrados sea fluida (Gido, 2003). Dicha situación se observó en la cooperativa pequeña y podría pensarse que dicho modelo de comunicación es difícil de reproducir en organizaciones de mayor tamaño.

No obstante, el punto medular no estriba en la cantidad de colaboradores de la organización, o en los medios tecnológicos de los cuales disponen para realizar el planteamiento, gestión o monitoreo de los proyectos de innovación. Existen a criterio de los autores dos elementos fundamentales: el factor cultural o clima de la organización, y el modelo de gestión de las comunicaciones que presenta la organización Prusak (1997). Estos dos elementos deben estar sincronizados para permitir que las ideas innovadoras tengan un trato ágil y eficaz antes, durante y posterior a su implementación en la organización; de manera que, los involucrados directos y toda la organización se sientan parte de la iniciativa en todo momento.

Diseño del modelo de negocio. Cada organización debe de ajustarlo a su modelo de negocio, y debe responder al planteamiento estratégico de la misma. Por tanto, si la estrategia está alineada a los planes de innovación, entonces la estructura deberá de responder a los esquemas operativos para la gestión de la innovación.
Tal caso se observó en el sujeto de estudio cooperativa mediana. Dadas las condiciones cambiantes del mercado eléctrico en Costa Rica, para la recta final del primer decenio del siglo XXl, se requiere que las organizaciones se transformen para acoplarse a las nuevas condiciones del mercado, eso es antropológicamente conocido como un proceso de adaptación natural y supervivencia. Dicha consideración es de suma importancia pues, la estructura funcional de la organización tiene un impacto directo sobre la velocidad de gestión de las innovaciones en la misma. Las organizaciones con estructuras planas tienen mayor velocidad de respuesta que las estructuras jerárquicas piramidales tradicionales en los modelos de gestión de los años setentas.

Es preciso que la estructura empresarial considere la innovación y su sistema de gestión como el motor para la creación de conocimiento y procesos de mejora en la organización. No existe innovación sin un conocimiento precedente, afirma Gutiérrez (2007); no obstante, es posible ampliar el alcance del concepto y decir que tampoco existe sin una estructura operativa que lo sostenga y estimule. Entonces, se requiere que las organizaciones migren a estructuras operativas que permitan el desarrollo eficiente de los Sistemas de Gestión de la Innovación.

Sistema de gestión del conocimiento. Tal situación permite incursionar en un tercer concepto o buena práctica identificada en la investigación desarrollada por la Sociedad Anónima Municipal. Dicha organización decidió iniciar en el 2006 los primeros intentos para la creación de un Sistema de gestión del conocimiento. Para el periodo bajo estudio del sujeto (2007), la organización ya había desarrollado algunos esquemas que perfilaban un modelo empresarial para el desarrollo y gestión de las iniciativas de innovación.

Tal situación fue bien favorable y es un caso de éxito por considerar, pues en el 2008 permitió a la organización concretar un esfuerzo de años anteriores y lograr el Premio a la Excelencia Empresarial, según Soto (2006), de la Cámara de Industrias de Costa Rica. El desarrollo y puesta en marcha del Sistema de Gestión, aún a un nivel primitivo, permitió a este sujeto de estudio organizar su actividad empresarial con respecto al inicio, planificación, desarrollo, cuantificación, control y seguimiento, beneficios, lecciones aprendidas y 
cierres de los diferentes proyectos de innovación que desarrollaba. Esta situación permitió la documentación de conocimiento que hasta antes no era aprovechado por otros colaboradores en la organización.

Catálogo de innovaciones. El uso de dicha herramienta desarrollada para realizar la identificación, cuantificación, clasificación y alcance de las iniciativas de innovación de una organización, permite al gestor de innovación contar con conocimiento valioso para el desarrollo de nuevos proyectos y mejora de los costos (Dzul, 2008) y rendimientos de estos. La estructura de la herramienta permite considerarla como una ficha técnica la cual ayuda en el registro de parámetros importantes, los cuales podrán ser evaluados para determinar que tan atractiva podría ser la iniciativa de acuerdo con la experiencia que tiene la organización, o en su defecto que consideraciones y salvedades debe realizar para una gestión exitosa de estas.

\section{Recomendaciones}

Dada la actual situación que presenta el sector de distribución de energía eléctrica con relación con la gestión de las iniciativas y procesos de innovación, es preciso que la alta dirección incorpore la gestión de estas como mecanismo integral del accionar de la organización. Dicha situación propiciará que los colaboradores no miren la innovación como algo extraño, reservado para las unidades de Investigación, Desarrollo e Innovación ( $\mid+D+1)$, sino como labores cotidianas que aportan al plan estratégico. Esta es una práctica que puede ser reproducida en otros sectores y organizaciones a nivel internacional.

Las organizaciones bajo estudio, deben iniciar la elaboración de un registro o portafolio de iniciativas innovadoras. Además, es preciso realizar una reforma profunda en cuanto a estructura, modelo, estándar o metodología de trabajo formal y sistemática para las innovaciones generadas por cada organización, pues a la fecha han sido desarrolladas bajo un sistema Adhoc sin una misma base metodológica. Es necesario que toda organización inicie por la adquisición de la información sobre la operación de su propio negocio. Es preciso que las organizaciones del sector de distribución de energía eléctrica, comprendan que la innovación no es un gasto, por el contrario, es un costo operativo.
Esta primera investigación exploró el estado actual de madurez en cuanto a la sistematización de los procesos y proyectos de innovación en las organizaciones. Para ello se utilizó un nuevo modelo de madurez denominado La Pirámide Invertida. A partir de la información recabada sería interesante para futuras investigaciones estudiar las fuentes que originan dichos estados y esquemas de gestión, así como el impacto que este rezago innovador ha tenido para otros sectores productivos y para la economía del país.

\section{Bibliografía}

Bernal, A. (2000). Metodología de la Investigación para administración y economía. Editorial Prentice Hall. Bogotá, Colombia. pp. 53-233. ISBN: 958-699-002-8.

Bravo B., Dzul L. \& Gracia V., S. (2009). Coordinación entre los niveles de gestión de proyectos: portafolio, programa y proyecto. DYNA Ingeniería e Industria, 84(5), 7I-78.

COTEC. (1998). TEMAGUIDE: Pautas Metodológicas en Gestión de la Tecnología y de la Innovación para Empresas. http://www.cotec.es/publicaciones/publicaciones.cfm [Obtenido el 10 de mayo del 20l0].

Dzul, L. \& Gracia V., S. (2008). Los costes de la calidad en el diseño de proyectos de construcción: un enfoque de procesos. DYNA Ingeniería e Industria, 83(I ), 4 I I-422.

Funiber. (2006). Curso Gestión del Conocimiento y Aprendizaje Organizacional. Fundación Iberoamericana Universitaria. España. www.funiber.org. [Obtenido el 10 de mayo del 2009].

Gido, J \& Clements, J. (2003). Administración Exitosa de Proyectos. $2^{\mathrm{a}}$.ed. Thomson Learning. México D.F. México. 396 pp. ISBN: 0-324-07| 68-X.

Goodstein L., et.al. (1998). Planeación Estratégica Aplicada. Editorial McGraw - Hill. Colombia. pp. I09-I39. ISBN: 958-600-706-5.

Gutiérrez, William. (2007). Gestión de Proyecto para I+D +l. En: Actas II Congreso Centroamericano de Administración de Proyectos, PMI. Memoria del Congreso. Heredia, Costa Rica 21-23 de Setiembre del 2007. http://www.pmi-costarica.org/apcon/ [Obtenido ell 0 de octubre del 2007].

Hernández, R.; Fernández, C \& Batista, P. (1998). Metodología de la Investigación. Editorial McGraw - Hill, Bogota. pp. 63-7| 9. ISBN: 970- | 0-5753-8.

ISO. (2002). The ISO Survey of ISO 9000 and ISO 14000 certificates. Eighth cycle. International Organization for Standardization. http://www.iso.org/iso/iso_catalogue/ catalogue_tc/catalogue_detail.htm?csnumber $=46486$ [Obtenido el I0 de mayo del 20I0]. 
Kaplan, R. \& Norton, D. ( 1992). Cuadro de Mando Integral. Editorial Mc Graw Hill. México. pp. 213-305. ISBN: 958-42- I 106-4.

Kluyver, A. (200 I). Pensamiento Estratégico, una perspectiva para los Ejecutivos. Editorial Prentice Hall. I ${ }^{\text {a }}$.ed., Buenos Aires, Argentina. p. I - 66. ISBN: 987-9460-59-6.

Muñoz, C. (1998). Cómo elaborar y asesorar una investigación de tesis. PrenticeHall Hispanoamericana. México D.F. México. pp. 108-|36. ISBN: 970-17-0|39-9.

Ohmae, K. (1982). The Mind of the Strategist: The Art of the Japanese Business. I ' . ed. Mc Graw Hill. USA. pp. 9- 136. ISBN- I 0: 0070475954.
Porter, M. ( 1986). ¿Qué es Estrategia competitiva?. Revista INCAE, Vol. X, No I, I 997. pp. 35-52.

Project Management Institute, PMI. (2004). Una Guía de los Fundamentos de la Administrador de Proyectos. 3 a .ed. PMI Publications. Pennsylvania, USA. pp. I-38. ISBN: |-930699- | 8-2.

Prusak, L. ( 1 997). Knowledge in Organizations. ButterworthHeinemann, EEUU. p. I I-30. ISBN: 0750697 I 80.

Soto, P. (2006). Informe Premio a la Excelencia. Anotaciones para informe. Informe Inédito. Heredia [s.n.], ESPH, Heredia, Costa Rica. Informe técnico de la Dirección de Investigación y Desarrollo de la ESPH. 